\title{
Multiple Adaptive Neuro-Fuzzy Inference Systems for Accurate Microwave CAD Applications
}

\author{
Juan Hinojosa and Ginés Doménech-Asensi \\ Departamento de Electrónica, Tecnología de Computadoras y Proyectos \\ Universidad Politécnica de Cartagena \\ Cartagena, Spain \\ E-mail: \{juan.hinojosa, gines.domenech\}@upct.es
}

\begin{abstract}
An approach for applying fuzzy logic for accurate CAD of microwave circuits is presented. Our proposed method combines space-mapping (SM) technology and multiple adaptive neuro-fuzzy inference systems (MANFIS) for the modeling of microwave devices. MANFIS is trained to predict a nonlinear vector multidimensional mapping function, which is obtained from SM approach. Optimization by micro-genetic algorithm is used to find nonlinear vector multidimensional mapping function for singular systems. This approach is applied to a shielded microstrip line within a region of interest. The parameter values $\left(\varepsilon_{\text {reff }}(f), Z_{c}(f)\right)$ computed with our proposed method are in excellent agreement with those obtained from electromagnetic simulations.
\end{abstract}

\section{INTRODUCTION}

In [1], a group of experts estimated the state of microwave computer-aided design (CAD) in the year 2010. In a panel discussion, R. Sorrentino predicted new algorithms or hybrid methods will be developed to increase the computational efficiency of the microwave CAD. In this sense, we propose an innovate hybrid approach with the aim to extract accurate and computationally efficient device models easy to be integrated into any CAD environment.

The method described in this paper uses multiple adaptive neuro-fuzzy inferences systems (MANFIS) [2-3] to model mapping functions obtained from space-mapping (SM) technology [4-8]. SM defines a framework for optimization of an accurate and expensive non-linear model (fine model) by using a less accurate and fast model (coarse model), through parameters mapping. Once the mapping function is found, the coarse model (CM) with the mapped parameters can be used to model quickly the microwave device to nearly the same degree of accuracy as that afforded by the fine model (FM). On the other hand, MANFIS is an extension of ANFIS to produce multiple real responses of the target system. It can model nonlinear functions of arbitrary complexity without employing precise quantitative analyses. Moreover, the knowledge acquired by the fuzzy inference system during the training with stipulated input-output data pairs can be extracted by means of Takagi and Sugeno's if-then rules [9].
In contrast to a set of weights that stores the knowledge in artificial neural network (ANN) used in different neural spacemapping approaches [5-7], these fuzzy if-then rules are readable, easy to be understood by human beings, and simple to be integrated in any CAD environment.

The feasibility of using fuzzy logic systems in microwave CAD area has been demonstrated in [10]. However, the method presented in this last work is not centered to the device modeling using space-mapping technology. To demonstrate the application of our hybrid modeling technique, a shielded microstrip line has been employed as illustration example. An initial coarse model is proposed in Section II. Section III presents the approach exploiting MANFIS and space-mapping technology. The results of the space-mapped neuro-fuzzy model obtained within a region of interest for the shielded microstrip line are provided in Section IV. Finally, conclusions are presented in Section V.

\section{CoArse Model of THE ShIElded Microstrip LiNE}

A shielded microstrip line (Fig. 1) has been used as illustration example for showing the validity of the hybrid modeling method described in this paper.

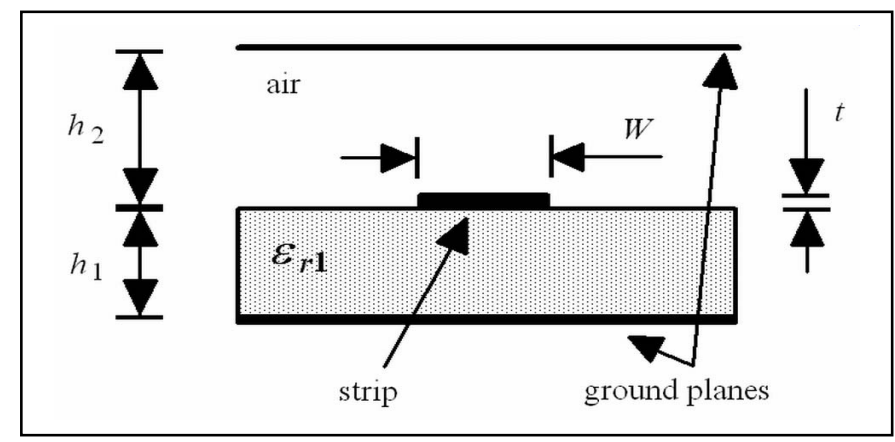

Figure 1. Cross section of the shielded microstrip transmission line.

An initial coarse model (CM) of this device can be obtained from Schwarz-Christoffel's transformation [11] and Getsinger's function [12]: 


$$
\begin{gathered}
\varepsilon_{\text {reff }}(0)=\left(\varepsilon_{r 1} \frac{K\left(k_{1}^{\prime}\right)}{K\left(k_{1}\right)}+\frac{K\left(k_{2}^{\prime}\right)}{K\left(k_{2}\right)}\right) /\left(\frac{K\left(k_{1}^{\prime}\right)}{K\left(k_{1}\right)}+\frac{K\left(k_{2}^{\prime}\right)}{K\left(k_{2}\right)}\right), \\
Z_{c}(0)=60 \pi /\left(\sqrt{\varepsilon_{r e f f}(0)}\left(\frac{K\left(k_{1}^{\prime}\right)}{K\left(k_{1}\right)}+\frac{K\left(k_{2}^{\prime}\right)}{K\left(k_{2}\right)}\right)\right), \\
\varepsilon_{r e f f}(f)=\varepsilon_{r 1}-\frac{\varepsilon_{r 1}-\varepsilon_{r e f f}(0)}{1+G\left(f / f_{p}\right)^{2}} \\
Z_{c}(f)=Z_{c}(0) \sqrt{\varepsilon_{r e f f}(0) / \sqrt{\varepsilon_{r e f f}(f)}}
\end{gathered}
$$

where $G=0.6+0.009 Z_{c}(0)$ and $f_{p}=Z_{c}(0) /\left(2 h_{1} \eta_{0} \sqrt{\mu_{0} \varepsilon_{0}}\right)$. The parameters $k_{1}, k_{2}, k_{1}^{\prime}$ and $k_{2}^{\prime}$ are the bounds of the shielded microstrip line structure:

$$
\begin{gathered}
k_{1}=\left[\cosh \left(\frac{\pi W}{4 h_{1}}\right)\right]^{-1}, \\
k_{2}=\left[\cosh \left(\frac{\pi W}{4 h_{2}}\right)\right]^{-1}, 1 \leq \frac{h_{2}}{h_{1}} \leq 5, \\
k_{2}=\left[\cosh \left(\frac{\pi W}{10 h_{2}}\right)\right]^{-1}, \frac{h_{2}}{h_{1}}>5, \\
k_{1}^{\prime}=\sqrt{1-k_{1}^{2}} \text { and } k_{2}^{\prime}=\sqrt{1-k_{2}^{2}} .
\end{gathered}
$$

$K\left(k_{1}\right), K\left(k_{1}^{\prime}\right), K\left(k_{2}\right)$ and $K\left(k_{2}^{\prime}\right)$ are the complete first order elliptical integrals of modulus $k_{1}$ and $k_{2}$ and complementary modulus $k_{1}^{\prime}$ and $k_{2}^{\prime}$. These integrals are calculated from analytical relationships [13].

Large errors in calculations are expected with this CM, since some assumptions were realized for SchwarzChristoffel's transformation and the dispersion expression is only valid for microstrip lines. Our contribution consists in showing that the hybrid modeling technique described in the next Section in conjunction with this CM can be applied for extracting an accurate space-mapped neuro-fuzzy model.

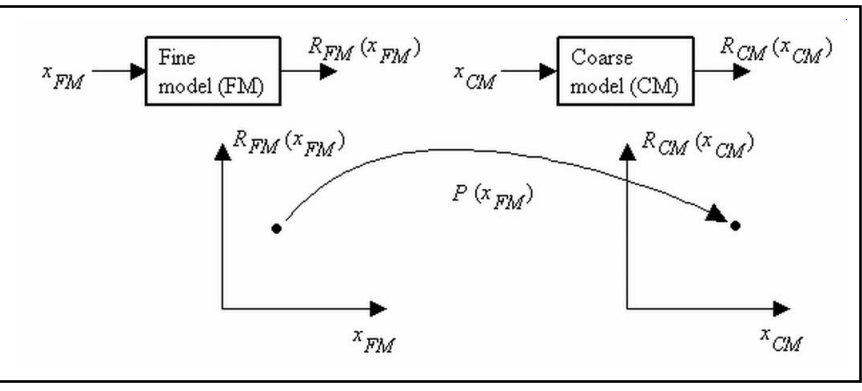

Figure 2. Space-mapping concept.

\section{Proposed Modeling Method}

In this Section, the space-mapping (SM) concept [4] is briefly described. The application of multiple adaptive neurofuzzy inference systems (MANFIS) in conjunction with the SM approach for the modeling of microwave devices is then presented.

\section{A. SM Concept}

The space-mapping (SM) concept requires a coarse model (CM) (ideal, fast, or low fidelity) and a fine model (FM) (accurate, practical, or high fidelity). As depicted in Fig. 2, the $\mathrm{CM}$ and FM design parameters are denoted by $x_{C M}$ and $x_{F M}$, respectively. The corresponding response vectors are denoted by $R_{C M}$ and $R_{F M}$, respectively. The thought of the SM consists in finding a mapping $P$ relating the FM and CM parameters within a region of interest as [4]:

$$
x_{C M}=P\left(x_{F M}\right),
$$

such that: $\quad R_{C M}\left(P\left(x_{F M}\right)\right) \approx R_{F M}\left(x_{F M}\right)$.

Once the mapping $P$ is found, the coarse model with the mapped parameters can be used for fast and accurate simulations.

\section{B. SM-Based Neuro-fuzzy Modeling}

In our approach, the mapping $P$ from the FM input-space to CM input-space is implemented by multiple adaptive neurofuzzy inference system (MANFIS) as illustrated in Fig. 3. It takes into account the frequency (freq) together with the physical parameters vector $(x)$ to train the MANFIS and to produce a mapped vector $P(x$, freq $)$ on the physical parameters (Fig. 3a). The number $m$ of ANFIS is equal to the number $m$ of input parameters (vector $x$ ) to be mapped. The structure of the $k$ th ANFIS is the same as (11) and (12) suggested by Takagi and Sugeno [2, 9]:

$$
\begin{aligned}
R_{k}^{i}: & \text { if } x_{1} \text { is } A_{1, k}^{i} \text { and } x_{2} \text { is } A_{2, k}^{i}, \cdots, x_{m} \text { is } A_{m, k}^{i}, \\
& \text { and freq is } A_{m+1, k}^{i} \text { then } \\
& y_{k}^{i}=a_{m+1, k}^{i} \text { freq }+a_{m, k}^{i} x_{m}+\cdots+a_{1, k}^{i} x_{1}+a_{0, k}^{i},
\end{aligned}
$$




$$
\hat{y}_{k}=\left(\sum_{i=1}^{c} w_{k}^{i} \cdot y_{k}^{i} / \sum_{i=1}^{c} w_{k}^{i}\right)
$$

with

$$
w_{k}^{i}=A_{1, k}^{i}\left(x_{1}\right) \times \cdots \times A_{m, k}^{i}\left(x_{m}\right) \times A_{m+1, k}^{i}(\text { freq }),
$$

where $R_{k}^{i}(i=1,2, \cdots, \mathrm{c}$ and $k=1,2, \cdots, m) \quad$ represents the $i$ th fuzzy rule of the $k$ th ANFIS. $x_{j}(j=1, \cdots, m)$ are the input physical parameters, freq is the frequency vector and $y_{k}^{i}$ the output of the fuzzy rule $R_{k}^{i}$. $A_{j, k}^{i}$ 's are linguistic labels, which are assigned to the corresponding Gaussian membership functions (with two parameters identified by premise parameters). The variables $a_{j, k}^{i}$ are the consequent parameters. $\hat{y}_{k}$ is the real output of the $k$ th ANFIS, which corresponds to the mapping $\left(P_{k}(x\right.$, freq $\left.)\right)$ of the input parameter $x_{k}$. In this paper, the number $\left(n_{g}\right)$ of Gaussian membership functions associated to each input is equal to the number $m$ of input parameters plus the frequency: $n_{g}=m+1$.

The number $c$ of fuzzy rules is equal to $c=(m+1)^{m+1}$ and it is the same for each ANFIS.

Mapping $P$, which can be a nonlinear multidimensional vector, can be found by solving the optimization problem:

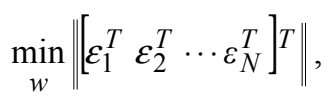

where vector $w$ (Fig. 3) contains the optimized premise and consequent parameters of the $m$ ANFIS's, which require $c \times m$ fuzzy rules (11). $N$ is the total number of training data and $\varepsilon_{K}$ is the error vector:

$$
\varepsilon_{K}=\mid R_{F M}\left(x_{I}, \text { freq }_{J}\right)-R_{C M}\left(P\left(x_{I}, \text { freq }_{J}\right), \text { freq }_{J}\right) \mid,(15
$$

with $I=1, \cdots, B P, J=1, \cdots, F P$ and $K=J+F P(I-1) . B P$ is the number of training base points for the input design parameters. $F P$ is the number of frequency points per frequency sweep. The total number of training data is $N=B P \times F P$. The premise and consequent parameters of each ANFIS are obtained during the training (Fig. 3a), using a hybrid learning algorithm which combines the least square method and the gradient method [2].

The optimization problem (14) is solved either by a gradient descent method (as example) or an optimization procedure based on a micro-genetic algorithm ( $\mu \mathrm{GA})$ [14] when this one is nonsingular or singular, respectively. In the case of many local optima, the mapping $P$ is found by minimizing the error vector (15) using the following error criterion (fitness required in $\mu \mathrm{GA}$ ):
Fitness $=1-\Delta_{i}$,

with

$$
\Delta_{i}=\sqrt{\varepsilon_{K_{-} i}}=\sqrt{\left|\begin{array}{l}
R_{F M}\left(x_{I}, \text { freq }_{J}\right)- \\
R_{C M, i}\left(P\left(x_{I}, \text { freq }_{J}\right)_{i}, \text { freq }_{J}\right)
\end{array}\right|},
$$

$$
\begin{aligned}
& P\left(x_{I}, \text { freq }_{J}\right)_{i}=\left[\begin{array}{lll}
\hat{y}_{1, I_{-} i} & \hat{y}_{2, I_{-} i} \cdots \hat{y}_{m, I_{-} i}
\end{array}\right]^{T} \\
& =\left[P_{1_{-} i}\left(x_{I}, \text { freq }_{J}\right) \cdots P_{m_{-} i}\left(x_{I}, \text { freq }_{J}\right)\right]^{T},
\end{aligned}
$$

where $i$ is the number of generation and $P_{k_{-} i}\left(x_{I}, f r e q_{J}\right)$ is the randomly generated solution for the input parameter $x_{k}$, Ith training base point and $J$ th frequency point. The best solution is identified at each iteration and error (15) is calculated. This error is checked against a predefined value. If it is satisfied, the simulator saves the result and passes to the following training base point up to finish with the total number $N$ of training data.

Once the mapping $P$ is found for the total number $N$ of training data and once the MANFIS is trained (Fig. 3a), a space-mapped neuro-fuzzy model (Fig. 3b) for fast and accurate simulations of the implicated device is immediately available within a region of interest.

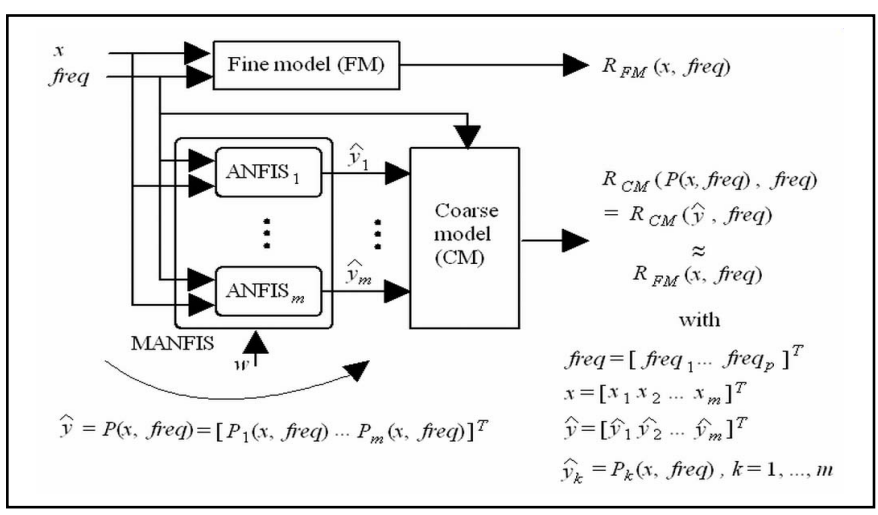

(a)

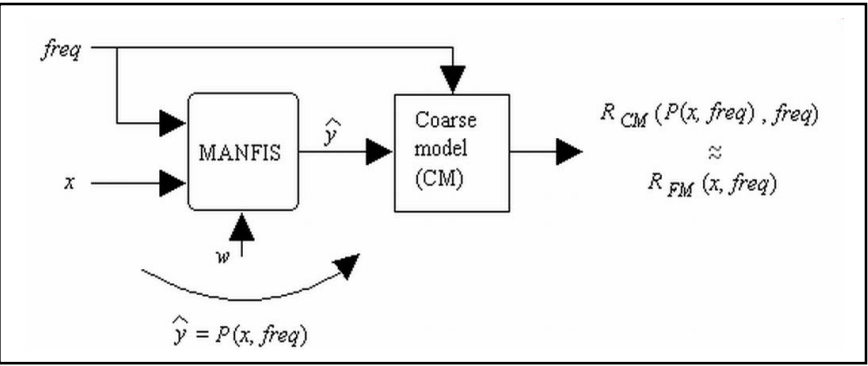

(b)

Figure 3. Hybrid approach combining SM and MANFIS. (a) Training the MANFIS to approximate the FM response. (b) Designing with the already trained MANFIS 


\section{RESULTS}

The above-described modeling approach was applied to the shielded microstrip line (Fig. 1). CM and FM data (2400) within the region of interest shown in Table I were provided, respectively, from analytical relationships presented in Section II and spectral domain approach (SDA) [15]. Some parameters were fixed: $t=0 \mu m$ and $h=254 \mu m$. The total number of input parameters with the included frequency is equal to 4 . The outputs of the CM and FM are the effective permittivity $\left(\varepsilon_{\text {reff }}(f)\right)$ and the characteristic impedance $\left(Z_{c}(f)\right)$. The modeling technique presents a singular system. Thus, a microgenetic algorithm $(\mu \mathrm{GA})$ as optimization method was applied to find the nonlinear multidimensional vector mapping function. The parameters used by the $\mu \mathrm{GA}$ were 30 for the number of chromosomes per individual, 5 for the population size, 100 for the maximum number of generations and $10^{-4}$ for the predefined error value. Crossover and mutation probability values were fixed to 0.5 and 0.02 , respectively. On the other hand, half of data (1200) exploring the whole of the region of interest (Table I) were used for training MANFIS (Fig. 3a) and the other half (1200) for testing (Fig. 3b). 3 epochs were required to train the modeling technique (Fig. 3a).

Mean and max relative errors over the coarse model (CM) and the space-mapped neuro-fuzzy model (SM-MAMFIS) for desired outputs $\left(\varepsilon_{\text {reff }}(f), Z_{c}(f)\right)$ with respect to expected ones (FM test data, which were different to those used for training) are shown in Table II. As expected, CM presents large errors. They are due to the assumptions realized to obtain the quasi-static model and to the dispersion expression which is not valid for the shielded microstrip line. On the other hand, SM-MANFIS model improves considerably the results of the $\mathrm{CM}$, showing an excellent agreement with those obtained from EM simulations with SDA. The CPU time for a single structure and one frequency point, on Pentium4-based computer, is lower than 100 milliseconds for the SMMANFIS model, while it takes more than 15 seconds for the EM simulation from SDA.

\section{CONCLUSION}

This paper presents an approach in which multiple adaptive neuro-fuzzy inference systems (MANFIS) and spacemapping (SM) technology are used to accurately model a shielded microstrip line. The extracted space-mapped neurofuzzy model combines a coarse model (CM) with trained MANFIS from mapped parameters. This model has the capability to compute parameters nearly as accurate as those obtained from full-wave electromagnetic (EM) simulations. The computation time is negligible with regard to other fullwave EM techniques. Therefore, this computational speed makes the coarse model (CM) with the trained MANFIS from mapped parameters suitable for interactive CAD applications. Although the example demonstrated only transmission line electrical parameters modeling, the method may be applied to other microwave devices.
TABLE I. REGION OF INTEREST FOR THE SHIELDED MiCROSTRIP LINE

\begin{tabular}{|l|c|c|c|}
\hline Parameters & Minimum Value & Maximum Value & Step \\
\hline$h_{2} / h_{1}$ & 1 & 3 & 1 \\
\hline$W / h_{1}$ & 0.8 & 3 & 0.1 and 1 \\
\hline$\varepsilon_{r 1}$ & 12 & 15 & 1 \\
\hline$f$ & $1 \mathrm{GHz}$ & $40 \mathrm{GHz}$ & $1 \mathrm{GHz}$ \\
\hline
\end{tabular}

TABLE II. RELATIVE ERRORS OVER CM AND SM-MANFIS WITH RESPECT TO FM

\begin{tabular}{|l|c|c|c|c|}
\hline \multirow{2}{*}{ Model } & \multicolumn{2}{|c|}{$\mathcal{E}_{\boldsymbol{r} \text { eff }}$ (relative error \%) } & \multicolumn{2}{c|}{$\boldsymbol{Z}_{\boldsymbol{c}}$ (relative error \%) } \\
\cline { 2 - 5 } & Mean & Max & Mean & Max \\
\hline CM & 9.81 & 46.5 & 14.45 & 39.56 \\
\hline SM-MANFIS & 0.033 & 0.31 & 0.045 & 0.60 \\
\hline
\end{tabular}

\section{REFERENCES}

[1] R. C. Bootin, et al., "Microwave CAD in the year 2010 - A panel discussion," Int J. of RF and Microw. Comp.-Aided-Eng., vol. 9, pp. 439-448, Oct. 1999.

[2] J.-S. R. Jang, "ANFIS: Adaptive-network-based fuzzy inference system," IEEE Trans. On Syst., Man, And Cybernetic, vol. 23, pp. 665685, May/June 1993.

[3] J.-S. R. Jang, C.-T. Sun, and E. Mizutami, Nuero-fuzzy and soft computing: A computational approach to learning and machine intelligence, Prentice Hall, New Jersey, 1997.

[4] J. W. Bandler, R. M. Biernacki, S. H. Chen, P. A. Grobelny, and R. H. Hemmers, "Sapce mapping technique for electromagnetic optimization," IEEE Trans. Microw. Theory Tech., vol. 42, pp. 25362544, Dec. 1994.

[5] J. W. Bandler, M. A. Ismail, J. E. Rayas-Sánchez, and Q. J. Zhang, "Neuromodeling of microwave circuits exploiting space mapping technology," IEEE Trans. Microwave Theory Tech., vol. 47, pp. $2417-$ 2427, Dec. 1999.

[6] M. H. Bakr, J. W. Bandler, M. A. Ismail, J. E. Rayas-Sánchez, and Q. J. Zhang, "Neural space-mapping optimization for EM-based design," IEEE Trans. Microwave Theory Tech., vol. 48, pp. 2307-2315, Dec. 2000.

[7] J. W. Bandler, M. A. Ismail, J. E. Rayas-Sánchez, and Q. J. Zhang, "Neural inverse space mapping (NISM) optimization for EM-based microwave design," Int. J. RF Microwave Computer-Aided Eng., vol. 13, pp. 136-147, 2003.

[8] J. W. Bandler, Q. S. Cheng, S. A. Dakroury, M. H. Bakr, K. Madesn, and J. Søndergaard, "Sapce mapping: The state of the art," IEEE Trans. Microw. Theory Tech., vol. 52, pp. 337-361, Jan. 2004.

[9] T. Takagi, and M. Sugeno, "Fuzzy identification of systems and its applications to modeling and control," IEEE Trans. On Syst., Man, And Cybernetic, vol. 15, pp. 116-132, Jan./Feb. 1985.

[10] V. Miraftab, and R. R. Mansour, "Computer-aided tuning of microwave filters using fuzzy logic," IEEE Trans. Microw. Theory Tech., vol. 50, pp. 2781-2788, Dec. 2002.

[11] R. E. Collin, Field theory of guided waves, 2nd ed., IEEE Press, New York, 1976, pp. 259-273.

[12] W. J. Getsinger, "Microstrip dispersion model," IEEE Trans. Microwave Theory Tech., vol. 21, pp. 34-39, Jan. 1973.

[13] W. Hilberg, "From approximations to exact relations for characteristic impedances," IEEE Trans. Microwave Theory Tech., vol. 17, pp. 259265, May 1969

[14] K. Krishnakumar, "Mcro-genetic algorithms for stationary and nonstationary function optimization," in Proc. SPIE Conf. On Intelligence and Adaptive Systems, vol. 1196, Phil, PA, pp. 289-296, 1989.

[15] T. Itoh, and R. Mittra, "Spectral-domain approach for calculating the dispersion characteristics of microstrip lines," IEEE Trans. Microw. Theory Tech., vol. 21, pp. 496-499, July 1973. 